\title{
Qualidade de vida de crianças pré-escolares e sua relação com a cárie dentária e fatores sociodemográficos
}

Quality of life of preschool children and their relationship with dental caries and sociodemographic factors Calidad de vida de los niños de preescolar y su relación con caries dentales y factores sociodemográficos

Faldryene de Sousa QUEIROZ ${ }^{1}$

Maria Luísa de Assis BRAGA ${ }^{2}$

Tuanny Lopes Alves SILVESTRE ${ }^{3}$

Édila Pablizia Cavalcante BATISTA ${ }^{3}$

Luciana Ellen Dantas COSTA ${ }^{1}$

Eliete Rodrigues de ALMEIDA ${ }^{4}$

${ }^{1}$ Professora Doutora do curso de Odontologia na UFCG - Centro de Saúde e Tecnologia Rural, Universidade Federal de Campina Grande (UFCG) 58708-110, Patos - PB, Brasil

${ }^{2}$ Graduanda em Odontologia na UFCG - Centro de Saúde e Tecnologia Rural, Universidade Federal de Campina Grande (UFCG) 58708-110, Patos - PB, Brasil

${ }^{3}$ Graduada em Odontologia na UFCG - Centro de Saúde e Tecnologia Rural, Universidade Federal de Campina Grande (UFCG) 58708-110, Patos - PB, Brasil

${ }^{4}$ Professora Doutora do Curso de Odontologia da UNICSUL - Departamento de Odontologia, Universidade Cruzeiro do Sul, 01506-000, São Paulo - SP, Brasil

\section{Resumo}

Objetivo: Investigar a relação entre cárie dentária, fatores socioeconômicos e a qualidade de vida de pré-escolares. Metodologia: A amostra foi composta por 215 crianças da cidade de Patos-PB. A prevalência da cárie dentária foi avaliada pelo índice ceo-d e os questionários Early Childhood Oral Health Impact Scale (B-ECOHIS) e o Autoquestionnaire Qualité de Vie Enfant Imagé (AUQEI) avaliaram o impacto da cárie na qualidade de vida das crianças. Os dados foram submetidos ao teste do Qui-quadrado, com nível de significância de 5\%. Resultados: Observou-se prevalência de cárie em $65,1 \%$ da amostra com média de ceo- $d=3,53$, com $92,6 \%$ do componente cariado. A cárie dentária apresentou relação significante com alguns fatores socioeconômico $(p<0,05)$ e o impacto na qualidade de vida das crianças foi observado $(p=0,001)$. Alguns quesitos específicos do AUQEI apresentaram relação com a cárie dentária: "à noite, ao dormir" $(p=0,01)$, "quando pratica esporte" $(p=0,047)$, "quando brinca sozinho" $(p=0,049)$ e "quando os amigos falam de você" $(p=0,044)$. Conclusão: Conclui-se que a cárie dentária influenciou negativamente na qualidade de vida das crianças e o conhecimento desses dados possibilita adequação e reorientação dos cuidados direcionados à melhoria das condições de saúde e, consequentemente, da qualidade de vida.

Descritores: Cárie Dentária; Fatores Socioeconômicos; Qualidade de Vida; Saúde Bucal.

\section{Abstract}

Objective: To evaluate the association between dental caries, socioeconomic conditions and life quality in preschoolers. Methodology: The cross-sectional study was conducted with 215 children of Patos city (PB). The prevalence of dental caries was evaluated using the DMFT index and the Early Childhood Oral Health Impact Scale (B-ECOHIS) questionnaires and the Autoquestionnaire Qualité de Vie Enfant Imagé (AUQEI) assessed the impact of caries on children's quality of life. The data were submitted to the Chi-square test, with a significance level of $5 \%$. Results: It was found a prevalence of $65.1 \%$ of caries and 3.53 of DMFT, with $92.6 \%$ of decayed teeth. The impact of dental caries on the quality of life of children examined was observed $(p=0,001)$. The individual assessment of the questions of the AUQEI has shown statistically significant relationship in the following items: "at night, at bedtime" $(p=0,01)$ "when practicing sport" $(p=0.047)$, "when playing alone" $(p=0.049)$ and "when friends talk about you" ( $p=0.044)$. Conclusion: It was concluded that dental caries negatively influenced the quality of life of children, knowledge of these data enables adjustment and reorientation of care aimed at improving the health and consequently the child's life quality.

Descriptors: Dental Caries; Socioeconomic Factors; Quality Of Life; Oral Health.

\section{Resumen}

Objetivo: investigar la relación entre la caries dental, los factores socioeconómicos y la calidad de vida de los preescolares. Metodología: La muestra consistió en 215 niños de la ciudad de Patos-PB. La prevalencia de la caries dental se evaluó utilizando el índice ceo-d y los cuestionarios de la Escala de impacto de la salud bucal en la primera infancia (B-ECOHIS) y el Autoquestionnaire Qualité de Vie Enfant Imagé (AUQEI) evaluó el impacto de la caries en la calidad de vida de los niños. Los datos se sometieron a la prueba de Chi-cuadrado, con un nivel de significación del $5 \%$. Resultados: La prevalencia de caries se observó en el $65,1 \%$ de la muestra con una media de $d m-d=3,53$, con el $92,6 \%$ del componente cariado. La caries dental mostró una relación significativa con algunos factores socioeconómicos $(p<0.05)$ y se observó el impacto en la calidad de vida de los niños $(p=0.001)$. Algunas preguntas específicas de la AUQEI estaban relacionadas con la caries dental: "de noche, cuando duerme" ( $p=0.01)$, "cuando hace deporte" ( $p=0.047)$, "cuando juega solo" $(p=0.049)$ y " cuando los amigos hablan de ti " $(p=0.044)$. Conclusión: Se concluye que la caries dental tuvo una influencia negativa en la calidad de vida de los niños y el conocimiento de estos datos permite la adecuación y la reorientación de la atención dirigida a mejorar las condiciones de salud y, en consecuencia, la calidad de vida.

Descriptores: Caries Dental; Factores Socioeconómicos; Calidad de Vida; Salud Bucal.

INTRODUÇÃO

A qualidade de vida relacionada a saúde está ligada ao impacto do estado de saúde sobre a capacidade do indivíduo de viver plenamente ${ }^{1}$. O interesse pela qualidade de vida e saúde bucal em crianças vem aumentando, uma vez que as desordens orais apresentam efeito negativo na qualidade de vida das mesmas ${ }^{2-5}$. Os problemas bucais podem causar dor, desconforto, dificuldades para mastigar, falar e outras condições que afetam a vida social, o exercício de atividades diárias e o bemestar do indivíduo $0^{6,7}$.

Ao longo dos anos, a saúde bucal tem sido avaliada a partir de critérios clínicos, os quais não permitem analisar o impacto dos problemas bucais na rotina dos indivíduos ${ }^{8} \mathrm{e}$, por este motivo, tem-se observado a incorporação de instrumentos de avaliação da 
qualidade de vida relacionada à saúde bucal (QVRSB) nos estudos sobre cárie dentária ${ }^{2}$, contribuindo para 0 planejamento $e$ implementação de estratégias de saúde bucal ${ }^{4,9}$.

Os fatores socioeconômicos têm se mostrado determinantes na condição de saúde bucal e consequentemente na qualidade de vida. Estudos revelaram que a prevalência de cárie dentária, expressada pelo índice ceo-d, mostrou-se significativamente pior para populações de baixo nível socioeconômico ${ }^{4,10-12}$. Grupos populacionais mais pobres, com baixa escolaridade e menor inserção no mercado de trabalho tendem a apresentar maior prevalência de cárie dentária, uma vez que a condição socioeconômica determina 0 acesso a atividades preventivas, visitas regulares ao dentista, escovação dentária e uma alimentação mais balanceada ${ }^{10}$.

A implementação de políticas públicas visando minimizar a desigualdade social através da expansão do acesso à saúde, educação, moradia, trabalho e lazer é essencial, quando se objetiva a obtenção de uma boa qualidade de vida $^{7,13,14}$.

Deste modo, considerando a relevância dos problemas bucais sobre o bem-estar da criança, o objetivo deste estudo epidemiológico transversal foi investigar a relação entre cárie dentária, condições socioeconômicas e qualidade de vida de pré-escolares.

MATERIAL E MÉTODO

O presente estudo foi aprovado no Comitê de Ética em Pesquisa com Seres Humanos da Universidade Cruzeiro do Sul sob protocolo CE/UCS- 140/2013. A pesquisa classificou-se como observacional transversal, no qual, o tamanho da amostra foi obtido por meio do processo de amostragem para população finita, segundo Cochran ${ }^{15}$. A margem de erro fixada e valor admitido para média e desvio-padrão foram os mesmos adotados pelo projeto SB Brasil $2003^{16}$ para a região Nordeste, para crianças de 5 anos de idade, em população maior de 100 mil habitantes. Nestas condições o tamanho amostral ficou estimado em 212 escolares. Admitindo-se um absenteísmo da ordem de $20 \%$, o tamanho final da amostra foi de 254 crianças.

A população alvo deste estudo foi composta por pré-escolares de ambos os sexos, matriculados em creches públicas e privadas, com 5 anos de idade, residentes em zona urbana e rural assim como, por seus respectivos pais/responsáveis, os quais receberam e assinaram o Termo de Consentimento Livre e Esclarecido (TCLE). Foram excluídas da amostra crianças que apresentaram deficiências neuropsicomotoras e/ou aquelas que não permitiram a realização do exame clínico, ou forem vetados pelos pais/responsáveis.

Antes da realização do estudo, realizouse um processo de calibração dos examinadores para a coleta dos índices ceo-d e aplicação dos questionários. A consistência dos diagnósticos foi medida através do coeficiente Cohens's kappa para a obtenção dos valores de concordância, os dados obtidos pelo teste de Kappa na calibração interexaminadores variou de 0,81-0,91 e na calibração intraexaminadores de 0,83-0,90, representando boa confiabilidade.

Um estudo-piloto foi realizado com a finalidade de avaliar os instrumentos de pesquisa e a dinâmica de coleta dos dados, para verificar as dificuldades observadas durante a realização do estudo, no preenchimento dos questionários e exame clínico, possibilitando assim, realizar os devidos ajustes. Para avaliação da condição de saúde bucal, adotou-se 0 índice ceo-d para o diagnóstico de cárie dentária ${ }^{17}$. Para avaliação da qualidade de vida dos pré-escolares, foi utilizado o instrumento Early Childhood Oral Health Impact Scale (ECOHIS) ${ }^{18}$, o qual foi inteiramente respondido pelos responsáveis das crianças. O ECOHIS foi desenvolvido a partir de uma seleção de 13 itens, oriundos dos 36 que compõem o questionário Child Oral Health Quality of Life Instrument (COHQOLI), esses itens foram considerados os mais relevantes para mensurar o impacto dos problemas bucais sobre a qualidade de vida dos pré-escolares e consideram as experiências de doenças bucais e tratamentos dentários da vida inteira das crianças.

Esse instrumento, na sua versão brasileira (B-ECOHIS), é composto por treze itens que avaliam o impacto dos problemas bucais sobre a criança (subescala da criança), sendo uma questão relativa a sintomas, domínio das limitações com cinco questões, duas relacionadas a fatores psicológicos, uma questão de autoimagem e uma de interação social. As outras quatro questões avaliam o impacto dos problemas bucais das crianças sobre sua família (subescala da família), sendo duas questões de angústia dos pais e duas questões de função familiar.

As opções de resposta estão elencadas em códigos que variam de 0 a 5 , onde o código $0=$ nunca, 1 = quase nunca, 2 = às vezes, $3=$ com frequência, $4=$ com muita frequência e $5=$ não sei.

Os escores totais e por domínios foram calculados a partir da somatória dos códigos das respostas. As respostas "não sei" foram 
contabilizadas, porém foram excluídas da somatória para o cálculo dos escores total e por domínios de cada entrevistado. A pontuação mínima obtida do questionário é zero, que corresponde a saúde bucal sem influencia na qualidade de vida dos pré-escolares e a máxima é 56 , onde existe forte influência da saúde bucal na qualidade de vida das crianças.

A somatória total da pontuação do questionário foi dividida em: sem impacto, quando o resultado da soma dos resultados foi igual a zero; impacto fraco, quando a soma da pontuação total foi maior que zero e menor ou igual a 18,67; impacto médio quando a somatória foi maior que 18,67 e menor ou igual a 37,34; e, impacto forte quando a soma da pontuação do questionário foi maior que 37,34 e menor ou igual a $56^{18}$. Para classificação socioeconômica do núcleo familiar foram avaliados o nível de escolaridade do chefe da família, a renda mensal familiar ${ }^{19}$, o número de membros da família que participam e /ou dependem da referida situação econômica ${ }^{20}$, o tipo de escola ${ }^{10}$, a condição de $\operatorname{moradia}^{19}$ e a localização do domicílio.

$\mathrm{O}$ instrumento utilizado nesta pesquisa para avaliar a qualidade de vida da criança, respondido pela própria criança, foi 0 Autoquestionnaire Qualité de Vie Enfant Imagé (AUQEI). Cada criança foi entrevistada individualmente, em um local reservado, no próprio ambiente escolar, por um único pesquisador devidamente calibrado.

A escala AUQUEI foi desenvolvida por Manificat e Dazord ${ }^{21}$, traduzida e validada por Assumpção $\mathrm{Jr}$ et $\mathrm{al}^{22}$ para o português é composta por 26 perguntas estruturadas em forma de escala com quatro possibilidades de respostas (muito feliz, feliz, infeliz e muito infeliz) e imagens representativas que refletem os sentimentos dos mesmos frente a conflitos que possam sofrer no cotidiano como: no relacionamento com familiares, amigos, profissionais da saúde e demais pessoas de seu convívio pessoal; comportamento apresentado nos ambientes escolar e hospitalar, nas refeições, brincadeiras e momentos de entretenimento; conhecimento e percepção de sua autoimagem; desempenho acadêmico e físico na prática de esportes; e suas perspectivas futuras.

Cada resposta recebeu uma pontuação de 0 a 3 , sendo o primeiro correspondente ao estado muito infeliz e o último ao estado muito feliz. O somatório dos escores de todas as questões representa o total individual, podendo este valor variar de 0 a 78. Após obtenção dos escores totais, a nota de corte utilizada foi de
48, sendo a qualidade de vida considerada prejudicada nos casos em que a pontuação for inferior a esta nota ou satisfatória nas crianças com escores iguais ou superiores a mesma.

Das 26 perguntas que compõem originalmente o questionário, 18 podem ser agrupadas em quatro domínios sendo esta divisão realizada da seguinte maneira: cinco questões $(1,2,4,5$ e 8) relacionadas às atividades na escola, refeições, ao deitar-se, e à ida ao médico formam o Domínio Função; outras cinco questões $(3,10,13,16$ e 18) relativas à opinião das crianças quanto às figuras parentais e delas, quanto a si mesmo constituem o Domínio Família; três questões $(11,21$ e 25) que abordam às férias, aniversário e relações com avós foram inseridas no Domínio Lazer; por fim, cinco questões $(15,17$, 19, 23 e 24) que se referem à independência, relacionamento com amigos e avaliação escolar constituem o Domínio Autonomia. As questões $6,7,9,12,14,20,22$ e 26 foram consideradas isoladamente, pois representam domínios separados dos demais.

Os dados foram analisados e processados por meio de planilhas Excel (®Microsoft 2013). A análise estatística foi realizada através de testes de Qui-quadrado, t de Student (ic=95\%, $p<0,05$ ). Os dados foram analisados através do software estatístico $R$ for Windows, versão 3.1 .0 e seus pacotes Hmisc versão 3.14-4 (2013-07-10), gplots versão 2.14.1.

RESULTADOS

- Fatores sociodemográficos e perfil da cárie dentária

O total de pares de crianças e seus respectivos pais/responsáveis participantes desse estudo foi de 215, correspondendo a $84,6 \%$ do total selecionado mediante a realização do cálculo amostral. A perda de 39 amostras ocorreu devido a: resposta incompleta do questionário $(74,7 \%)$, ausência da criança na escola/creche no dia de realização do exame clínico (18\%), e falta de colaboração por parte das crianças na realização do exame (7,3\%).

Das 215 crianças participantes, a maioria pertencia ao sexo masculino $(54,9 \%)$. Os préescolares apresentaram idade média de 63,5 meses ( $D P=3,55)$, variando de 60 a 71 meses, $71,6 \%$ eram alunos de escolas/creches públicas e $28,4 \%$ alunos de escolas particulares.

Avaliando a condição de saúde bucal, observou-se prevalência de cárie $=65,1 \%$ e índice ceo-d médio $=3,53 \quad(\mathrm{dp}=3,94$; cariado $=92,6 \%$,extraido $=4,7 \%$, obturado $=2,7 \%$ ), sendo que $35,8 \%$ das crianças apresentavam 1 a 5 dentes cariados e $29,3 \%$ mais de 6 . 
A Tabela 1 mostra a prevalência de cárie dentária em relação às variáveis de ordem sociodemográficas, pelo teste Qui-Quadrado $(p<0,05)$. Verificou associação estatisticamente significante entre a presença de cárie e a renda mensal familiar $(p=0,004)$, entre a presença de cárie e o tipo de escola da criança $(p=0,001)$ e entre a presença de cárie e o tipo de moradia $(p=0,006)$.

Tabela 1. Correlação entre a presença de cárie dentária e os fatores sociodemográficos, Patos-PB, Brasil - 2020

\begin{tabular}{|c|c|c|c|c|}
\hline \multicolumn{5}{|c|}{ Cárie Dentária } \\
\hline Variável & $\begin{array}{c}\text { Ausente } \\
\mathrm{n}(\%)\end{array}$ & $\begin{array}{c}\text { Presente } \\
\mathrm{n}(\%)\end{array}$ & $\begin{array}{l}\text { Total } \\
\mathrm{n}(\%) \\
\end{array}$ & Valor de $\mathbf{p}^{*}$ \\
\hline \multicolumn{5}{|l|}{ VARIÁVEIS CRIANÇAS } \\
\hline \multicolumn{5}{|l|}{ Sexo } \\
\hline Masculino & $42(35,6)$ & $76(64,4)$ & $118(100,0)$ & \multirow{2}{*}{0,462} \\
\hline Feminino & $33(34,0)$ & $64(66,0)$ & $97(100,0)$ & \\
\hline \multicolumn{5}{|c|}{ VARIÁVEIS SOCIOECONÔMICAS } \\
\hline \multicolumn{5}{|c|}{ Escolaridade chefe família } \\
\hline Analfabeto & $13(34,2)$ & $25(65,8)$ & $38(100,0)$ & \multirow{5}{*}{0,468} \\
\hline $\begin{array}{ll}\text { Ensino } & \text { fundamental } \\
\text { incompleto } & \end{array}$ & $10(28,6)$ & $25(71,4)$ & $35(100,0)$ & \\
\hline Ensino fundamental completo & $24(29,6)$ & $57(70,4)$ & $81(100,0)$ & \\
\hline Ensino médio & $19(43,2)$ & $25(56,8)$ & $44(100,0)$ & \\
\hline Ensino superior & $8(47,1)$ & $9(52,9)$ & $17(100,0)$ & \\
\hline \multicolumn{5}{|l|}{ Renda mensal familiar } \\
\hline$<1$ salário mínimo & $27(30,3)$ & $62(69,7)$ & $89(100,0)$ & \multirow[b]{2}{*}{0,004} \\
\hline $\begin{array}{l}\text { 1-2 salário mínimo } \\
>3 \text { salários }\end{array}$ & $\begin{array}{l}32(31,1) \\
15(62,5)\end{array}$ & $\begin{array}{l}71(68,9) \\
8(34,8)\end{array}$ & $\begin{array}{l}103(100,0) \\
23(100,0)\end{array}$ & \\
\hline \multicolumn{5}{|c|}{ Quantidade de pessoas no domicílio } \\
\hline$<6$ pessoas & $65(34)$ & $126(66)$ & $191(100,0)$ & \multirow{2}{*}{0,470} \\
\hline$\geq 6$ pessoas & $9(37,5)$ & $15(62,5)$ & $24(100,0)$ & \\
\hline \multicolumn{5}{|l|}{ Tipo de escola } \\
\hline Pública & $43(27,9)$ & $111(72,1)$ & $154(100,0)$ & \multirow[b]{2}{*}{0,001} \\
\hline Privada & $31(50,8)$ & $30(49,2)$ & $61(100,0)$ & \\
\hline \multicolumn{5}{|l|}{ Zona do domicílio } \\
\hline Rural & $11(26,8)$ & $30(73,2)$ & $41(100,0)$ & \multirow{2}{*}{0,260} \\
\hline Urbana & $63(36,2)$ & $111(63,8)$ & $174(100,0)$ & \\
\hline \multicolumn{5}{|l|}{ Tipo de Moradia } \\
\hline Própria & $25(26,6)$ & $69(73,4)$ & $94(100,0)$ & \multirow{4}{*}{0,006} \\
\hline Financiada & $9(75)$ & $3(25)$ & $12(100,0)$ & \\
\hline Alugada & $32(39,0)$ & $50(61,0)$ & $82(100,0)$ & \\
\hline Cedida & $8(29,6)$ & $19(70,4)$ & $27(100,0)$ & \\
\hline
\end{tabular}

*teste Qui-quadrado / Significante ao nível de 5,0\%

$\mathrm{Na}$ Tabela 2 observa-se a distribuição do percentual de dentes cariados, com extração indicada e restaurados segundo a renda mensal familiar e tipo de escola da criança, onde verificou associação estatisticamente significante entre os componentes do ceo-d e o tipo de escola. $\mathrm{Na}$ avaliação da razão de chances $(\mathrm{RC})$ da presença de cárie dentária para exposição à escola pública, foi encontrada uma razão de 2,67 para um (IC $95 \%=1,45$ 4,92 ), ou seja, existem 2,67 possibilidades da cárie ocorrer no grupo de crianças que estudam em escola pública contra uma de ocorrer no grupo de crianças que estudam em escolas particulares.

Tabela 2. Distribuição do percentual de dentes cariados, com extração indicada e restaurados segundo a renda mensal familiar e tipo de escola da criança, Patos-PB, Brasil - 2020

\begin{tabular}{|c|c|c|c|c|}
\hline \multicolumn{5}{|c|}{ Componentes do ceo-d } \\
\hline \multirow{2}{*}{ Variável } & Cariado & Perdido & Obturado & \multirow{2}{*}{$\begin{array}{c}\mathbf{p}^{*} \\
\mathbf{R C} / \mathbf{I C}\end{array}$} \\
\hline & $\mathrm{n}(\%)$ & $\mathrm{n}(\%)$ & $\mathrm{n}(\%)$ & \\
\hline \multicolumn{5}{|c|}{ FATORES SOCIOECONÔMICOS } \\
\hline \multicolumn{5}{|c|}{ Renda mensal familiar } \\
\hline$<1$ salário mínimo & $352(50)$ & $28(77)$ & $7(35)$ & \multirow{3}{*}{0,475} \\
\hline $1-2$ salário mínimo & $330(47)$ & $8(23)$ & $13(65)$ & \\
\hline$\geq 3$ salários & $21(3)$ & $0(0)$ & $0(0)$ & \\
\hline \multicolumn{5}{|l|}{ Tipo de escola } \\
\hline Pública & $611(86,9)$ & $30(83,3)$ & $12(60,0)$ & \multirow{2}{*}{$\begin{array}{c}0,004 \text { RC } 3,65 \\
\text { (IC } 95 \% ; 1,45-4,92) \\
\end{array}$} \\
\hline Privada & $92(8,81)$ & $6(16,7)$ & $8(40,0)$ & \\
\hline
\end{tabular}

- Impacto da cárie dentária na qualidade de vida das crianças

Em relação aos dados obtidos por meio da aplicação do B-ECOHIS, que avalia a influência da saúde bucal na qualidade de vida da criança, 51,2\% mostraram qualidade de vida prejudicada devido à condição de saúde bucal, onde $45,1 \% \quad(n=97)$ apresentaram um impacto fraco da saúde bucal na qualidade de vida, $4,7 \% \quad(n=10)$ das crianças apresentaram um impacto médio da saúde bucal na qualidade de vida e 01 criança $(0,5 \%)$ apresentou forte impacto da saúde bucal na qualidade de vida.

A média dos escores por quesito podem ser observados na Tabela 3. Percebe-se que os itens "dor de dente" $(0,72)$, "responsável sentiuse culpado pela condição de saúde bucal do filho" $(0,44)$ e "dificuldade de comer devido a problema nos dentes" $(0,41)$, foram os que receberam pontuações mais altas, contribuindo negativamente para a qualidade de vida, enquanto que "evitou falar devido a problema nos dentes" $(0,07)$, "dificuldade em pronunciar palavras" $(0,08)$ e "impacto financeiro por tratamento dentário" $(0,1)$ foram os de menor pontuação.

$\mathrm{Na}$ sessão do impacto na criança as questões que demonstraram maiores médias foram "dor de dente" $(0,72)$, "dificuldade de comer devido a problema nos dentes" $(0,41)$, "dificuldade de dormir devido a problema nos dentes" $(0,38)$ e "dificuldade de beber devido a problema nos dentes" $(0,38)$ e na sessão de impacto na família as questões que demonstraram maiores médias foram "responsável sentiu-se culpado pela condição de saúde bucal do filho" $(0,44)$ e "responsável ficou aborrecido devido a problemas nos dentes do filho" $(0,33)$, conforme a Tabela 1 .

A Tabela 3 mostra ainda a distribuição por domínios do B-ECOHIS, onde se observou que na sub-escala Criança, os domínios de maior e menor média foram, respectivamente, o Domínio dos Sintomas $(0,72)$ e o Domínio da interação Social $(0,07)$ e na sub-escala Família, o domínio da Angústia dos Pais foi o que apresentou maior influência negativa na qualidade de vida $(0,38)$.

Em relação aos valores obtidos por meio da aplicação do AUQEI, a amplitude de pontuação variou de 15 a 78 (de um total de 78 pontos), com média de 52,1 (DP: 9,6). Observou-se que a maioria das crianças ( $n=145,67,4 \%$ ) obteve um escore maior que 48 , o que é classificado como uma qualidade de vida satisfatória (Tabela 4).

$\mathrm{Na}$ avaliação das médias dos escores por domínio, observou-se que o Domínio 
Autonomia foi 0 que mais influenciou negativamente na qualidade de vida $(1,67)$ e $o$ Domínio Lazer foi o que mostrou grau de satisfação mais elevado $(2,34)$.

Percebe-se que o perfil obtido das respostas mostrou grau de satisfação elevada para a maioria dos itens (maior que 1,5). Onde o item que apresentou maior satisfação foi "no dia do aniversário" $(2,51)$ "à mesa junto a família" $(2,44)$ e "quando assiste TV" $(2,34)$ e os de maior insatisfação foram "quando fica internado" $(1,03)$, "quando fica longe da família" $(1,09)$ e "quando vai ao médico" $(1,42)$.

Tabela 3. Média aritmética dos escores por quesito e domínios do B-ECOHIS, Patos-PB, Brasil - 2020

\begin{tabular}{|c|c|c|}
\hline Quesitos do B ECOHIS por domínio & $\begin{array}{l}\text { Média por } \\
\text { quesito* }^{*}\end{array}$ & $\begin{array}{c}\text { Média por } \\
\text { domínio }\end{array}$ \\
\hline \multicolumn{3}{|l|}{$\begin{array}{l}\text { SUB-ESCALA CRIANÇA } \\
\end{array}$} \\
\hline \multicolumn{3}{|l|}{ DOMÍNIO DOS SINTOMAS } \\
\hline 1.Sentiu dores nos dentes, na boca ou nos maxilares & 0,72 & 0,72 \\
\hline \multicolumn{3}{|l|}{ DOMÍNIO DAS LIMITAÇÕES } \\
\hline 2.Teve dificuldade em beber bebidas quente ou fria & 0,38 & \multirow{5}{*}{0,25} \\
\hline 3.Teve dificuldade para comer certos alimentos & 0,41 & \\
\hline 4.Teve dificuldade de pronunciar qualquer palavra & 0,08 & \\
\hline 5a.Já faltou à creche, jardim de infância ou escola & 0,19 & \\
\hline 5b.Deixou de fazer alguma atividade diária (brincar) & 0,20 & \\
\hline \multicolumn{3}{|l|}{ DOMÍNIO DOS ASPECTOS PSICOLÓGICOS } \\
\hline 6.Teve dificuldade em dormir & 0,38 & \multirow{2}{*}{0,37} \\
\hline 7.Já ficou irritada & 0,37 & \\
\hline \multicolumn{3}{|l|}{ DOMÍNIO DA AUTOIMAGEM } \\
\hline 8.Já evitou sorrir & 0,11 & 0,11 \\
\hline \multicolumn{3}{|l|}{ DOMÍNIO DA INTERAÇÃO SOCIAL } \\
\hline 9.Já evitou falar & 0,07 & 0,07 \\
\hline \multicolumn{3}{|l|}{$\begin{array}{l}\text { SUB-ESCALA FAMÍLIA } \\
\end{array}$} \\
\hline \multicolumn{3}{|l|}{ DOMÍNIO DA ANGÚSTIA DOS PAIS } \\
\hline 10.Ficou aborrecida & 0,33 & \multirow{2}{*}{0,38} \\
\hline 11.Já se sentiu culpada & 0,44 & \\
\hline \multicolumn{3}{|l|}{ DOMÍNIO DA FUNÇÃO FAMILIAR } \\
\hline 12.Já faltou ao trabalho & 0,17 & \multirow{2}{*}{0,13} \\
\hline 13.Já teve impacto financeiro por tratamento & 0,10 & \\
\hline \multicolumn{3}{|c|}{ *Média dos escores por quesito (0-nunca - 4- Com muita frequência) } \\
\hline \multicolumn{3}{|c|}{$\begin{array}{l}\text { Tabela 4. Média dos escores por domínio do AUQUEI, Patos-PB } \\
\text { Brasil - } 2020\end{array}$} \\
\hline AUQEI & \begin{tabular}{c|c}
$\begin{array}{c}\text { Média por } \\
\text { item* }\end{array}$ & \\
\end{tabular} & $\begin{array}{l}\text { Média por } \\
\text { Domínio }\end{array}$ \\
\hline \multicolumn{3}{|l|}{ DOMÍNIO FUNÇÃO } \\
\hline à mesa, junto com sua família & 2,44 & \multirow{5}{*}{1,98} \\
\hline à noite, quando você se deita & 1,93 & \\
\hline na sala de aula & 2,23 & \\
\hline à noite, ao dormir & 1,89 & \\
\hline quando você vai a uma consulta médica & 1,42 & \\
\hline \multicolumn{3}{|l|}{ DOMÍNIO FAMÍLIA } \\
\hline se você tem irmãos, quando brinca com eles & 2,06 & \multirow{5}{*}{2,11} \\
\hline quando você pensa no seu pai & 2,15 & \\
\hline quando você pensa na sua mãe & 2,19 & \\
\hline quando seu pai ou sua mãe falam de você & 1,98 & \\
\hline alguém te pede que faça algo que sabe & 2,17 & \\
\hline \multicolumn{3}{|l|}{ DOMÍNIO LAZER } \\
\hline no dia do seu aniversário & 2,51 & \multirow{3}{*}{ 2,34 } \\
\hline durante as férias & 2,28 & \\
\hline quando você está com seus avós & 2,21 & \\
\hline \multicolumn{3}{|l|}{ DOMÍNIO AUTONOMIA } \\
\hline quando você brinca sozinho (a) & 1,46 & \multirow{5}{*}{1,67} \\
\hline quando você dorme fora de casa & 1,74 & \\
\hline quando os amigos falam de você & 1,82 & \\
\hline quando você está longe da sua família & 1,09 & \\
\hline quando você recebe as notas da escola & 2,25 & \\
\hline
\end{tabular}

$\mathrm{Na}$ avaliação das médias dos escores por domínio, observou-se que o Domínio Autonomia foi 0 que mais influenciou negativamente na qualidade de vida $(1,67)$ e 0 Domínio Lazer foi o que mostrou grau de satisfação mais elevado $(2,34)$.

Percebe-se que o perfil obtido das respostas mostrou grau de satisfação elevada para a maioria dos itens (maior que 1,5 ). Onde o item que apresentou maior satisfação foi "no dia do aniversário" $(2,51)$ "à mesa junto a família"
$(2,44)$ e "quando assiste TV" $(2,34)$ e os de maior insatisfação foram "quando fica internado" $(1,03)$, "quando fica longe da família" $(1,09)$ e "quando vai ao médico" $(1,42)$.

A Tabela 5 apresenta a relação entre cárie dentária e qualidade de vida, baseada na classificação do B-ECOHIS, onde observou-se correlação entre a presença de cárie dentária e a influência da saúde bucal na qualidade de vida $(p<0,05)$. Analisando a razão de chances (RC/ IC 95\%) da influência da cárie dentária sobre a qualidade de vida, foi encontrada uma razão de 3,49 para um (IC 95\% = 1,92- 6,34), ou seja, existem 3,49 mais possibilidades de crianças com cárie dentária terem a qualidade de vida prejudicada, do que as crianças sem a presença da doença.

Tabela 5. Correlação entre experiência de cárie dentária e qualidade de vida pelo B-ECOHIS, Patos-PB, 2020.

\begin{tabular}{|c|c|c|c|c|c|c|}
\hline \multicolumn{7}{|c|}{ CÁRIE DENTÁRIA } \\
\hline Variável & Ausente & Presente & Total & \multicolumn{2}{|c|}{ RC (OR) } & IC 95\% \\
\hline \\
\hline Sem Impacto & $\frac{52(48,6)}{22(213)}$ & $\frac{55(51,4)}{85(78,7)}$ & $107(100,0)$ & 0,0001 & 3,49 & $1,92-6,34$ \\
\hline
\end{tabular}

Foi avaliado ainda a relação entre as respostas afirmativas aos quesitos do BECOHIS e a presença de cárie dentária, por meio do teste Qui-quadrado. Constatou-se uma rrelação estatisticamente significante entre ambos, em todos os quesitos, exceto: dificuldade em pronunciar palavras $(p=0,534)$, evitou sorrir $(p=0,107)$, responsável ficou aborrecido $(p=0,214)$ e impacto financeiro por tratamento $(p=0,257)$.

Ao avaliar a relação entre as respostas relacionadas ao sentimento de tristeza aos quesitos do AUQEI e a presença de cárie dentária, por meio do teste Qui-quadrado $(p<0,05) \quad$ constatou-se uma correlação estatisticamente significante entre ambos nos quesitos: "Como você se sente à noite ao dormir" $(p=0,01)$, "Como você se sente quando pratica esporte (brinca)" $(p=0,047)$, "Quando brinca só" $(p=0,049)$ e "Quando os amigos falam de você" $(p=0,044)$.

Quanto a relação entre qualidade de vida e condições socioeconômicas, a classificação da qualidade de vida da criança não apresentou associação significativa com nenhuma variável sociodemográfica.

DISCUSSÃO

A cárie dentária ainda é considerada um grave problema de saúde pública ${ }^{23}$. Embora exista uma diminuição na prevalência de cárie em crianças brasileiras, a mesma ainda se mantém alta em grupos específicos da população. Sendo estes caracterizados por uma menor renda, menor nível de educação e piores 
condições de higiene bucal, ou seja, há uma polarização da doença, com concentração e aumento da necessidade de tratamento em uma pequena parcela da população ${ }^{4,24}$.

Por esse motivo, neste estudo, optou-se por avaliar, associado à prevalência da cárie dentária, os dados sociodemográficos das famílias envolvidas e constatou-se que a renda mensal familiar, o tipo de moradia da criança e o tipo de escola que estuda apresentaram relação significativa com a presença de cárie dentária. Em uma revisão de literatura, Brizon et al. ${ }^{12}$ observaram que a maioria dos estudos analisados também apresentaram associação estatística significativa entre a prevalência de cárie dentária e os índices socioeconômicos, principalmente no tocante a escolaridade dos pais e renda familiar, dados também observados por Queiroz et al. ${ }^{4}$.

Constatou-se neste estudo, que as crianças apresentaram um ceo-d médio de 3,53 e que apenas $34,9 \%$ da população estudada estavam livres da cárie dentária, valores muito aquém das metas sugeridas pela Organização Mundial de Saúde ${ }^{17}$ para 2010, onde havia indicação de que $90 \%$ das crianças com 5 anos estivessem livres de cárie. $O$ estudo mais recente em âmbito nacional, o SB Brasil $2010^{25}$, encontrou uma média de ceo-d aos 5 anos de idade de 2,43, valor aquém ao encontrado neste estudo. No entanto, o valor do ceo-d nas cidades do interior do Nordeste foi de 3,94, corroborando com os valores obtidos na presente pesquisa.

Embora as crianças com maior renda mensal familiar e estudantes de escolas particulares apresentem maior probabilidade de estarem livres da cárie dentária, a avaliação da distribuição do percentual de dentes cariados, com extração indicada e obturados, segundo a renda familiar e o tipo de escola, demonstrou um alto percentual do componente não tratado da doença nas crianças enquadradas nestas categorias.

Com relação à distribuição dos componentes do ceo-d baseada no tipo de escola da criança, a maioria dos dentes restaurados eram em alunos de escolas públicas, em detrimento aos de escolas particulares, o que remetem uma certa negligência dos pais no controle da doença, ou mesmo a não valorização da dentição decídua, embora apresentem um poder aquisitivo maior e teoricamente maior acesso aos serviços de saúde. Os dados ainda podem sugerir que há bom funcionamento dos serviços de saúde pública local, facilitando o acesso à saúde da população de menor renda.
A alta prevalência de cárie dentária encontrada na população estudada pode estar relacionada a não fluoretação das águas de abastecimento público na localidade e ao baixo incentivo a políticas públicas preventivas em saúde, tendo um enfoque mais individual e abordagem tecnicista. A atenção preventiva e promocional deve ser realizada de maneira contínua e sistemática, podendo certamente elevar os níveis de saúde bucal da população assistida $^{26}$.

Mensurar qualidade de vida é um dever árduo, uma vez que a sua avaliação é muito subjetiva e de difícil quantificação. Nenhum instrumento ainda conseguiu abranger todas as informações necessárias ${ }^{22}$ e, por esse motivo, no presente trabalho, optou-se por utilizar dois instrumentos para avaliação da qualidade de vida infantil, diferentemente da maioria dos outros trabalhos ${ }^{4,7,14}$.

A escolha, especificamente, por esses dois instrumentos (AUQEI e B-ECOHIS), se deu devido ao primeiro ser respondido pela própria criança, ser de fácil entendimento e aplicação na faixa etária estudada e já ser traduzido e validado para utilização na língua portuguesa e por o B-ECOHIS ser respondido pelos pais, direcionado para avaliação da influência da saúde bucal na qualidade de vida da criança e da família e por ser o instrumento de escolha da maioria dos trabalhos nessa área ${ }^{6}$. Sendo, portanto, capaz de se obter no presente estudo, as duas percepções sobre da qualidade de vida da criança, a da própria criança e dos seus respectivos pais.

Apenas 9\% dos estudos realizados com crianças, envolveram avaliações feitas pelas próprias crianças. A avaliação do impacto dos agravos bucais a partir do relato da criança possibilita a compreensão de como ela se sente em relação à sua condição bucal, permitindo uma complementação da avaliação normativa realizada pelo profissional ${ }^{27}$.

A maioria opta por avaliar qualidade de vida por meio da resposta dos responsáveis. Crianças geralmente diferem dos adultos em relação à visão da qualidade de vida, enquanto os adultos a avaliam de uma forma mais prática, pela capacidade de viver independentemente, para as crianças esse fato pode estar relacionado a algo mais imaginário, como ter muitos amigos, correr no parque ou vestir roupas bonitas ${ }^{28}$.

Observou-se que a cárie dentária pode provocar um impacto negativo na vida da criança e de sua família, uma vez que o sentimento de dor, desconforto, dificuldades para dormir, se alimentar e irritação, foram os 
itens de maior impacto na qualidade de vida, baseada na avaliação do B-ECOHIS. No entanto, em relação à socialização, esta não foi afetada nas crianças do referido estudo, uma vez que o Domínio da Interação Social foi o que sofreu menor impacto em relação à condição de saúde bucal.

Algumas equivalências nas respostas aos dois questionários foram detectadas, confirmando a confiabilidade dos instrumentos utilizados. A dificuldade para dormir foi um dos itens de maior impacto na qualidade de vida baseada na avaliação do B-ECOHIS, corroborando com essa afirmativa, por meio da aplicação do AUQEI pôde-se perceber que os itens relacionados ao sono da criança: "quando deita" e "à noite, ao dormir", receberam uma das menores médias, permanecendo abaixo dos escores relacionados à satisfação (2- feliz; 3muito feliz), demonstrando o quanto a dor e o desconforto, ocasionado pela cárie dentária, interfere no sono das crianças, impactando negativamente na qualidade de vida. Estudos como os de Gradella et $\mathrm{al}^{29}$ e Nóbrega et. $\mathrm{al}^{3}$ confirmam tal afirmação.

Outro fator foi com relação a autoimagem, pôde-se observar que no BECOHIS esse foi o domínio de segundo menor impacto e na avaliação pelo AUQEI, o sentimento da criança ao ver uma foto sua, mostrou média satisfatória, com respostas oscilando entre feliz e muito feliz. Esse fator pode ser explicado pela pouca idade da população estudada, uma vez que, de acordo com a avaliação do desenvolvimento psicológico da criança, somente a partir dos 6 anos de idade é que se inicia a preocupação com a autoimagem e a comparação com outras crianças $^{30}$.

Observou-se que as crianças com presença de cárie dentária relataram sentiremse mais tristes durante realização de todas as atividades elencadas no questionário, com exceção de "como se sente no dia do aniversário", em detrimento das crianças livres de cárie. Entretanto, apenas as ações: "como você se sente à noite, ao dormir", "quando pratica esporte (brinca)", "quando brinca sozinho" e "quando os amigos falam de você" apresentaram relação estatisticamente significante.

Corroborando com esses achados, na avaliação, por quesito, do B-ECOHIS, constatou-se que as crianças com presença de cárie relataram um maior percentual de respostas afirmativas ao questionário em todos os quesitos, demonstrando um maior impacto na qualidade de vida nas crianças com cárie dentária. Observou-se associação estatisticamente significante entre a doença e a maioria dos quesitos, exceto: "dificuldade em pronunciar palavras", "evitou sorrir", "responsável ficou aborrecido" e "impacto financeiro por tratamento".

$\mathrm{Na}$ avaliação da qualidade de vida pelo B-ECOHIS, observou-se que o Domínio da Angustia dos Pais apresentou a segunda maior média, ficando atrás apenas do Domínio dos Sintomas, o que quer dizer que os pais se sentem culpados pela condição de saúde bucal dos seus filhos, no entanto, esses mesmos pais não tomam uma atitude de sanar essa doença, já que ela é passível de controle e socialmente evitável. Esse fato pode ser comprovado pela alta prevalência de cárie dentária encontrada nesse estudo, especificamente do componente cariado do ceo-d.

Outro dado importante e relacionado a esse tema seria com relação ao quesito "impacto financeiro" do B-ECOHIS, que apresentou a terceira menor média, afirmando que o orçamento dos pais não foi comprometido pelo problema de saúde bucal do filho, o que pode ser justificado pela não procura dos serviços de saúde, ou mesmo, ao fácil acesso aos serviços públicos.

No presente trabalho o Domínio dos Sintomas foi o que causou maior impacto na qualidade de vida, sendo a dor, um dos problemas mais frequentes nas crianças com cárie dentária, fato comprovado por Dias et al. ${ }^{5}$.

Não foi identificada relação estatisticamente significante entre os fatores sociodemográficos e a qualidade de vida em nenhum dos dois instrumentos utilizados. No entanto, pôde-se constatar que tanto as crianças, como seus familiares, apresentam a qualidade de vida comprometida em função das alterações bucais. Sabendo-se, portanto, que a condição de saúde bucal sofre bastante influência dos fatores sociodemográficos, como já abordado anteriormente, não se pode dissociar a avaliação da qualidade de vida das condições socioeconômicas.

CONCLUSÃO

Os resultados do presente estudo constataram uma alta prevalência de cárie dentária na população estudada, com percentual elevado do componente não tratado da doença. Observou-se correlação entre a ocorrência da cárie e os fatores socioeconômicos, além da forte influência da condição de saúde bucal na qualidade de vida da criança. O conhecimento desses dados possibilita uma adequação dos cuidados em saúde, assim como, uma reorientação dos 
gastos públicos, com atividades preventivas e assistenciais, direcionadas à melhoria das condições de saúde e, consequentemente, da qualidade de vida da criança.

\section{REFERÊNCIAS}

1. Gottardo AC, Webber LP, Rossa J, Arossi GA. Perda dental anterior influencia a qualidade de vida relacionada a saúde oral. Rev IC ULBRA 2015;13:60-70.

2. Eskenazi SEM, Sousa KG, Agostini LTP, Barbosa TS, Castelo PM. Avaliação da experiência de cárie e qualidade de vida relacionada à saúde bucal de escolares. Rev Bras Promoç Saúde. 2015;28(2):198-205.

3. Nóbrega AV, Moura LFAD, Andrade NS, Lima CCB, Dourado DG, Lima MDM. Impacto da cárie dentária na qualidade de vida de préescolares mensurado pelo questionário PedsQL Ciênc saude coletiva. 2019; 24(11):4031-41.

4. Queiroz FS, Costa LED, Silvestre TLA. Saúde bucal, fatores socioeconômicos e qualidade de vida de crianças de 12 anos de idade da cidade de Patos-PB. Arch Health Invest. 2018; 7(8):316-22.

5. Dias TKS, Ferreira GC, Almeida LHS. Cárie na primeira infância e qualidade de vida de pacientes de zero a 3 anos. Rev. UNINGÁ. 2019;56(S3):192-201.

6. Massarente DB, Domaneschi $\mathrm{C}$, Marques $\mathrm{HH}$, Andrade SB, Goursand D, Antunes JLF. Oral health-related quality of life of paediatric patients with AIDS. BMC Oral Health. 2011; 11(2).

7. Ramos-Jorge J, Pordeus IA, Ramos-Jorge ML, Marques LS, Paiva SM. Impact of untreated dental caries on quality of life of preschool children: different stages and activity. Community Dent Oral Epidemiol. 2014; 42(4):311-22.

8. Macedo A, Macena MCB, Rodriguez RQF. Autopercepção sobre saúde bucal dos usuários do restaurante popular de Patos/PB. Rev Saúde e Ciência On line. 2015;4(1):41-51.

9. Kumar S, Kroon J, Lalloo R. A systematic review of the impact of parental socio-economic status and home environment characteristics on children's oral health related quality of life. Health Qual Life Outcomes. 2014;12:41.

10. Piovesan C, Mendes FM, Ferreira FV, Guedes RS, Ardenghi TM. Socioeconomic inequalities in the distribuition of dental caries in Brazilian preschool children. J Public Health Dent. 2010; 70(4):319-26.

11. Figueiredo MC, Peixoto LT, Covatti $F$, Silva KVCL, Jardim LE. Saúde Bucal de Pessoas em Situação de Pobreza Extrema Residentes em um Município no Sul do Brasil. UNOPAR Cient Ciênc Biol Saúde. 2014;16(1):45-50.
12. Brizon VC, Melo RR, Zarzar PM, Gomes VE, Oliveira ACB. Indicadores socioeconômicos associados à cárie dentária: uma revisão crítica. Rev Unimontes Cient. 2014;16(1):79-91.

13. Marmot M. Social determonantes of health inequalities. Lancet. 2005;365(9464):1099-104.

14. Scarpelli AC, Paiva SM, Viegas CM, Carvalho AC, Ferreira FM, Pordeus IA. Oral healthrelated quality of life among Brazilian preschool children. Dent Oral Epidemiol. 2013;41(4): 336-44.

15. Cochran WG. Sampling Techniques. 3th ed. New York: Wiley; 1977.

16. Ministério da Saúde (BR). SB Brasil 2003. Brasília (DF); 2004.

17. Who. World Health Organization. Oral Health Suveys, basic methods. 4nd ed. Geneve; 1997.

18. Tesch FC, Oliveira BH, Leão A. Semantic equivalence of the Brazilian version of the Early Childhood Oral Health Impact Scale. Cad Saude Publica. 2008;24:1897-909.

19. Oliveira LB, Sheiham A, Bönecker M. Exploring the association of dental caries with social factors and nutritional status in Brazilian preschool children. Eur J Oral Sci. 2008; 116:37-43.

20. Melo MMDC, Souza WV, Lima MLC, Braga C. Fatores associados à cárie dentária em préescolares do Recife, Pernambuco, Brasil. Cad Saude Publica. 2011;27(3):471-85.

21. Manificat S, Dazord A. Évaluation de la qualité de vie de l'enfant: alidationd'un questionnaire, premiers résultats. Neuropsychiatr Enfance Adolesc. 1997;45(3):106-14.

22. Assumpção Jr FB, Kuczynski E, Sprovieri MH, Aranha EMG. Escala de Avaliação de Qualidade de Vida (AUQEI - Autoquestionnaire Qualité de Vie Enfant Imagé): Validade e confiabilidade de uma escala para qualidade de vida em crianças de 4 a 12 anos. Arq Neuropsiquiatr. 2000;58(1):119-27.

23. Rebelo STCP, Santanna GR. Prevalência de cárie dental em escolares de 12 anos na rede municipal de ensino de Parnaíba Piauí. Rev Interd Ciên Saúde. 2015;2(3):11-8.

24. Ramadan $\mathrm{YH}$, Koltermann AP, Piovesan C. Cárie dentária em crianças brasileiras: tendência e polarização. Disciplinarum Scientia. 2014;15(1):137-46.

25. Brasil. Ministério da Saúde. Secretaria de Atenção à Saúde. Secretaria de Vigilância em Saúde. SB Brasil 2010: Pesquisa Nacional de Saúde Bucal: resultados principais / Ministério da Saúde, 2012. Brasília: Ministério da Saúde.

26. Brasil. Ministério da Saúde. Secretaria de Atenção à Saúde. Política Nacional de Humanização. Formação e intervenção / Ministério da Saúde, Secretaria de Atenção à Saúde, Política Nacional de Humanização. Brasília: Ministério da Saúde, Cadernos Humaniza SUS. 2010.1:242. 


\section{CONFLITO DE INTERESSES}

Os autores declaram não haver conflitos de interesse

AUTOR PARA CORRESPONDÊNCIA

\section{Faldryene de Sousa Queiroz Feitosa}

Unidade Acadêmica de Ciências Biológicas, Centro de Saúde e Tecnologia Rural, UFCG - Universidade Federal de Campina Grande, Rod. PB-110, 58708-110, Jatobá, Patos - PB, Brasil

Telefone: (83) 3511- 3045

E-mail: falqueiroz@hotmail.com LB. Caries prevalence and severity, and quality of life in Brazilian 2- to 4-year-old children. Dent Oral Epidemiol. 2011;39(6):498-504.

30. Abanto J, Carvalho TS, Bönecker M, Ortega AO, Ciamponi AL, Raggio DP. Relatos dos pais da qualidade de saúde oral de vida de crianças Submetido em 16/06/2020 com paralisia cerebral. BMC Saúde Bucal. Aceito em 23/10/2020 2012. 